\title{
In Search for a Common Pathway for Health Issues in Men - the Sign of a Holmesian Deduction
}

\author{
Fouad Aoun*, Anthony Kallas Chemaly, Simone Albisinni, Marc Zanaty, Thierry \\ Roumeguère
}

\begin{abstract}
The evidence for the existence of a common pathway for health issues in men is presented in this review. Several epidemiological studies have shown that conditions like cardiovascular diseases (CVD), metabolic syndrome, diabetes, lower urinary tract symptom (LUTS), erectile dysfunction (ED), prostate cancer, hypogonadism, depression and suicide can be associated as risk factors for each other. Thus, the risk of CVD is significantly increased in men with metabolic syndrome, ED, hypogonadism, prostate cancer and/or LUTS. In addition, the above mentioned conditions are more prevalent in atherosclerotic patients. In addition, growing evidence indicates that low androgen levels can cause metabolic syndrome. In addition, obesity, dyslipidaemia and diabetes can further reduce androgen levels potentiating their adverse effect. Low testosterone levels are also associated with a higher incidence of aggressive prostate cancer on biopsy and on definitive pathology, and lower probability of abiraterone response in the metastatic setting. Several recent studies point towards diffuse endothelial dysfunction and dysregulated pro-inflammatory state as the biological link between all these disorders. Our current hypothesis is that oxidative stress caused by these dysfunctions explains the pathogenesis of each of these conditions.
\end{abstract}

Keywords: Epidemiology - men's health - atherosclerosis - erectile dysfunction - LUTS/BPH - hypogonadism - prostate

Asian Pac J Cancer Prev, 17 (1), 1-13

\section{Introduction}

Epidemiology is considered the basic science of public health and the term applied epidemiology is used to describe the application of epidemiology to address public health issues. In the last century, a key factor in advancing our understanding of health was the shift of interest from a whole population approach to a gendered based epidemiology. However, special attention was particularly paid toward women's health and it was only until the last decade that men's health received the same level of attention with many important issues being highlighted.

The leading men's health threat is considered to be cardiovascular disease (CVD) with $20 \%$ of men dying from a heart attack or a stroke and the average age of death is under 65 years (Thom et al., 2006). Men with diabetes have heart disease death rates about 2 to 4 times higher than adults without diabetes (Colombo et al., 2015). CVD and all-cause mortality are also increased in men with the metabolic syndrome, even in the absence of baseline CVD and diabetes (Colombo et al., 2015). Prostate cancer is another men's health issue and is considered as the leading cancer in men aged $>50$ years (Siegel et al., 2012). In $2012,14.1$ million men in the world were diagnosed with cancer and 8.2 million died from the disease (Siegel et al.,
2012). Men are also at risk of depression and suicide with a life time risk of $7 \%$ and a male to female ratio of 5.6 to 1 (Nock et al., 2008).

In addition, recently, non life threatening conditions that alter men's overall health had caught worldwide attention. In analogy to menopause, late onset hypogonadism gained popularity. Currently, ED and lower urinary tract symptoms due to bladder dysfunction and/or benign prostatic hyperplasia (LUTS/BPH) are considered as the most prevalent men's health problem. The low healthcare seeking rate and the potential detrimental effect on physiologic and mental health of these two conditions legitimate their incorporation in the main priorities of a good men's health.

It is of note that many of these conditions are age related and one could argue that the link between them is simply a result of their mutual association with increasing age. However, community and nationwide population based studies suggested that these conditions are independently associated with each other pointing instead to a causative relationship. From an epidemiological point of view, these associations should improve the understanding of the risk factors and the aetiology of these conditions, inform screening plans and certainly change counselling and treatment strategies. In the present report, the different associations between all these conditions 
are summarized, analysed and discussed. Emphasis will be placed on the observedpathophysiologic interactions linking these conditions to highlight the theory of a common pathogenic mechanism.

\section{Overwhelming Evidence of Links between ED, LUTS, and Metabolic Syndrome/CVD}

ED and CVD are known to be common age related changes in middle aged and older men with little variations in their prevalence among different countries. Several epidemiological studies have established that ED is frequent in men with a history of CVD (Gandaglia et al., 2014). According to these studies, the prevalence of ED in these patients varies between 47 and $75 \%$ (Vlachopoulos et al., 2013a). In addition, ED prevalence differs according to the severity, the extent and the clinical presentation of CVD. Montorsi et al. demonstrated that ED rate was lower in acute coronary syndrome and in one vessel disease compared to chronic coronary disease and multiple vessel lesions (22\% vs. 65\%, respectively) (Montorsi et al., 2006a). The severity and duration of ED was more related to the extent of atherosclerosis and to the number and severity of cardiovascular risk factor. The presence of ED in patients suffering from myocardial infarction, cerebrovascular events significantly increased the risk of mortality in two recent meta-analyses (Vlachopoulos et al., 2010; Vlachopoulos et al., 2013b). All risk factors for atherosclerosis are prevalent in patients suffering from ED and vice versa. Additionally, there is a significantly higher prevalence of men with metabolic syndrome having ED compared to healthy controls $(26.7 \%$ vs. $13 \%)$ (Esposito et al., 2005).

Available evidences ascertain that obesity, CVD, diabetes, and smoking represent established risk factors for ED (Rosen et al., 2004). Furthermore, ED preceded coronary artery events, stroke, and peripheral arterial disease by a significant period that usually ranges from 2 to 5 years (Jackson et al., 2010) which highlights its co-existence with occult CVD as evidence by a novel prospective angiographic study; $19 \%$ of ED patients presented an angiographically obstructed coronary artery disease (Vlachopoulos et al., 2005). Previous studies had reported an abnormal stress test in 5 to $56 \%$ of patients with ED (Montorsi et al., 2006b). The coronary angiography in these patients demonstrated obstructive coronary artery disease in $>90 \%$ of cases. These findings had led many clinicians to consider ED as an independent predictor for future cardiovascular events, especially in young patients and resulted in a paradigm shift in the classification and management of patients with ED (Nehra et al., 2012).

In a large cohort of men $(n=12825)$, the group suffering from ED had a 2-fold increase in the risk of acute myocardial infarction compared to potent men after adjusting for major risk factors except diabetes (Blumentals et al., 2004). These findings were confirmed in a prospective cohort of potent men in the Prostate Cancer Prevention trial (PCPT) (Anitha et al., 2009). Slightly more than half of these patients developed ED over a period of 5 years and after adjusting for all risk factors, ED was independently associated with an increased risk of acute coronary syndrome. The impact of ED was equal or higher than other cardiovascular risk factors. At present, organic ED is considered as a vascular disorder and acardiovascular risk factor in many patients.

\section{ED and LUTSs}

On the other hand ED is also associated with LUTS in both community and clinically based populations. This association is reported in single and multicentre as well in national and cross-national surveys or studies. In a large scale multinational survey conducted in the US and six European countries, 50\% of men aged 50-59 years with moderate LUTS had ED (Rosen et al., 2003). Conversely, sexual dysfunctions and their associated bother were strongly related to both age and severity of LUTS. Going from mild to moderate, or moderate to severe LUTS had a greater impact on ED than ageing by 10 years. The EpiLUTS study results also support the link between LUTS and ED and demonstrate an association between the severity of LUTS and ED (Wein et al., 2009). In the Cologne Male Survey, the prevalence of LUTS in men suffering from ED was $72 \%$, compared with $38 \%$ in potent men (Braun et al., 2000). Numerous systematic reviews of epidemiological studies conducted worldwide support the existence of a link between ED and LUTS that is independent of age and co-morbidities (Gacci et al., 2011; Seftel et al., 2013). Conversely, consistent correlation was found between the severity of ED at baseline and the incidence of LUTS or bother during follow-up. After adjustment for the confounders, the incidence rate ratio of LUTS was significantly higher in men with moderate or severe ED than in those free of ED at entry. Compared with men free of ED at baseline, the incidence rate ratio of urinary bother were 1.6 (95\% CI 1.1-2.4), 1.9 (95\% CI 1.1-3.2) and 2.2 (95\% CI 1.1-4.3) for minimal, moderate or severe ED, respectively (Shiri et al., 2007). Although LUTS represent a common syndrome, it is usually caused by benign prostatic hyperplasia (BPH) which affects $70 \%$ of men over the age of 70 years (Parsons, 2010). The BPH/ED clinic based studies demonstrated that LUTS and ED are highly prevalent in men with BPH (McVary, 2005). A recent systematic review representing 71322 men demonstrated the strong association between LUTS and ED with overall Odds Ratios varying from 1.4 to 9.74 (Bouwman et al., 2009). In a critical analysis of the literature published recently, 58-82\% of men with LUTS have ED and conversely $8-26 \%$ of men with ED have LUTS (Gacci et al., 2011).

These two conditions are strongly associated with a negative impact on the quality of life of ageing males and their partners and increase substantially the psychological burden on men's health (Kirby et al., 2013). This point is illustrated by several studies of female partners of men with ED or LUTS. In the FEMALES study, decreases in female sexual satisfaction and frequency of orgasm were significantly related to the male partner's self-reported severity of ED (Fisher et al., 2005). The multinational UrEpik study showed that the presence of LUTS in a patient had an adverse effect on the quality of life of the partner (Boyle et al., 2003). 


\section{LUTS and Metabolic Syndrome/CVD}

A few clinical studies have reported a cross-sectional and longitudinal relationship between LUTS and CVD. Furthermore, a convincing link between cardiovascular risk factors and BPH/LUTS is demonstrated by several recent studies. Hammarsten et al. found that patients with LUTS and individual components of metabolic syndrome were at increased risk to have larger prostate and faster annual BPH growth rates (Hammarsten et al., 1998). Similar findings were reported in a Turkish population (Ozden et al., 2007). A recent meta-analysis clearly indicated that metabolic syndrome is independently associated with increased prostate volume, in particular of the transitional zone and worse prognosis in older patient with a larger prostate $(>30 \mathrm{~mL}$ ) (De Nunzio et al., 2012). Wehrberger et al. demonstrated a significantly higher risk for current and future CVD and stroke in men with severe LUTS (Wehrberger et al., 2011). Ng et al. initiated a screening programme for cardiovascular risk factors in patients presenting with LUTS (Ng et al., 2007). They clearly demonstrated that men with moderate-to-severe LUTS had a statistically higher chance of having at least one cardiovascular risk factor during assessment. Similarly, a health screening project in Vienna reported an association between the severity of LUTS and the number of components of metabolic syndrome among 1724 men (Wehrberger et al., 2011). In the hallym aging study, Korean men with 3 or more vascular risk factors were 3 times more likely to have moderate/severe LUTS than men without vascular risk factors after controlling for age and body mass index (Kim et al., 2010).

In a cross-sectional large scale observational study representative of the US population, Rohrmann et al. demonstrated that men 60 years old or older with three or more components of the metabolic syndrome were at $80 \%$ increased risk for LUTS compared to those without any risk factor (Rohrmann et al., 2005). In contrast, in The Boston Area Community Health Survey, a similar association of LUTS and metabolic syndrome was shown in men younger than 60 years old (Kupelian et al., 2010). There are also some data that prostate volume and severity of LUTS increase with increasing waist circumference (Lee et al., 2012). Obese men are 30\% more likely to develop LUTS than their non-obese peers (Parsons et al., 2011). The burden of this metabolic syndrome was also demonstrated in the Olmsted County cohort study where patients treated by statins for dyslipidaemia had up to 7 year delay in the new onset of moderate and severe LUTS compared to patients without statins therapy ( $\mathrm{St}$ Sauver et al., 2011). Furthermore, the addition of statins therapy in a retrospective cohort decreased prostate volume up to 15 times (Lee et al., 2013). In the previous meta-analysis, HDL cholesterol appeared to be the major metabolic syndrome component responsible for prostate volume reduction (De Nunzio et al., 2012). Furthermore, vigorous physical activity was associated with up to $25 \%$ decrease in the risk of BPH/LUTS (Parsons and Kashefi, 2008). Even more interesting is the fact that patients not known to have any CVD who present with LUTS are at increased risk of subsequent hospitalisation for acute cardiovascular events, mainly stroke, as demonstrated by a novel study published recently (Lin et al., 2013). Lin et al. demonstrated, at an average follow up of 6.6 years and after adjusting for all covariates, significantly higher incidence rates of acute cardiovascular events and stroke in the LUTS group compared to the non LUTS group. In their population based representative cohort, hypertension, dyslipidaemia and diabetes were also more prevalent in the LUTS group. However, it is noteworthy to mention that few studies do not support this association. The majority of these studies are retrospective with a limited number of patients having significant LUTS. In a prospective observational population based study, Paans et al. did not found an association between LUTS and CVD but the authors focused their study on nycturia (Paans et al., 2011). A negative outcome was also reported in a Dutch registry study for the primary care setting (Bouwman et al., 2015).

From all the previous considerations, it can be concluded that LUTS, ED and CVD share some risk factors such as obesity, diabetes, hypertension, smoking, and ageing. In the largest cross-sectional multicentre survey on 3369 community-dwelling European men with a mean age of 60 years, these three conditions were clearly associated independently from other risk factor and demographic variables (Corona et al., 2010). In this sample, lower physical quality of life was associated with CVD, depression, LUTS, ED, obesity and hypertension. CVD, diabetes and smoking represented risk factors for ED even after adjustment for different centres. Countries with the lowest prevalence of ED reported the lowest prevalence of depression. LUTS and depression were also associated with orgasmic dysfunction (Corona et al., 2010).

\section{Clinical Evidence of Associations of Hypogonadism with ED, LUTS/BPH, Metabolic Syndrome and CVD}

Androgen deficiency increases as well with age; an annual decline in free testosterone, total testosterone and albumin bound testosterone of $2.8 \%, 1.6 \%$ and $2.5 \%$, respectively has been described (Camacho et

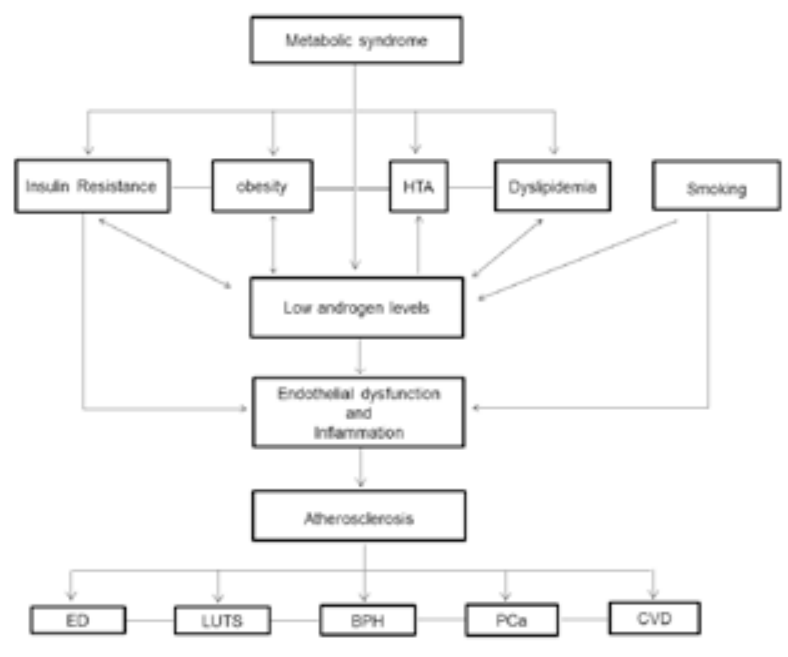

Figure 1. Pathophysiology of and Relationships between Common Health Issue's i n Men 
al., 2013). It is more prevalent in obese men, men with diabetes and in men with a poor health status or with comorbidities (Couillard et al., 2000; Kapoor et al., 2007). Hypogonadism is defined as a clinical syndrome that must comprise symptoms and biochemical confirmation with low circulating testosterone levels (Wang et al., 2009).

Androgens play a pivotal role in sexual function and androgens deficiency is a common cause of sexual dysfunction. Men with low testosterone levels have an increased risk of severe ED (Kalra et al., 2010). Conversely, ED is one of the most prevalent symptoms of male hypogonadism (Kumar et al., 2010; Isidori et al., 2014). One third of men with ED have androgen deficiency as demonstrated by the meta-analysis of Isidori et al. (Isidori et al., 2005). Corona et al. examined 803 patients and demonstrated that metabolic syndrome was associated with a more severe ED, which is further exacerbated by the coexistence of hypogonadism (Corona et al., 2006). As already mentioned hypogonadism and ED are prevalent in men with diabetes. A recent study found that the severity of ED correlates well with the level of testosterone in diabetic men (Kapoor et al., 2006). Interestingly, testosterone replacement therapy improves glycaemic control and lipid profile, sexual function, psychological and cognitive parameters, muscle mass and strength, bone marrow density and quality of life in these patients (Kapoor et al., 2006). Benefits include also a decrease in fat mass, body mass index and waist size (Kapoor et al., 2006). At an epidemiological level, this relationship is less clear and controversial for men with LUTS (Chang et al., 2009).

Furthermore, it is well known that androgens promote prostate growth rate and anti-androgen medications decrease prostate volume and LUTS in patients with BPH (Roehrborn et al., 2011). The association between testosterone and LUTS might be indirect and based on the link between LUTS and visceral obesity, the metabolic syndrome and ED. However, there are more positive and compelling data on testosterone replacement therapy in men with hypogonadism and LUTS (Karazindiyanoglu and Cayan, 2008; Pearl et al., 2013). In an exploratory study of a new form of long acting testosterone replacement therapy, a positive progressive effect on the IPSS over the study period was noted (Haider et al., 2009). A dose response effect was confirmed by two other studies published (Schatzl et al., 2000; Kenny et al., 2010). Men with hypogonadism and LUTS treated by testosterone replacement therapy experienced no worsening of their symptoms according to recent study (Pearl et al., 2013). Nearly one-third of those showed an AUA-SI improvement of more than 3 points. This positive outcome seems to be derived, most probably, from the effect of testosterone on bladder function. Despite an increase of $12 \%$ in volume of the prostate gland after 8 months of testosterone replacement treatment, Holmang et al. demonstrated an increase in peak urinary flow and mean urine volume voided in a testosterone-treated group of men compared to placebo treatment in a double-blind controlled study (Holmang et al., 1993). Similarly, in a double-blind placebo-controlled study, the authors demonstrated that normalizing serum androgen levels in aging men with hypogonadism had little effect on their
LUTS and urodynamic data (Sih et al., 1997).

In a retrospective study, Ko et al. found that men with moderate LUTS treated by TRT for a late onset hypogonadism maintained a relatively high maximal flow rate and improved both storage and voiding LUTS, without the clinical progression of BPH or rising PSA (Ko et al., 2013). Recently, a randomized control study demonstrated improvement in IPSS and maximal flow rate in the TRT group compared to placebo (Shigehara et al., 2011). In a prospective cohort of patients with hypogonadism treated by TRT, a significant increase in maximal bladder capacity and compliance, and decrease in detrusor pressure at maximal flow were noted (Karazindiyanoglu and Cayan, 2008). Testosterone injection in castrate rats demonstrated the same findings (Tek et al., 2010). An improvement in terms of the number of vessels, epithelial thickness, quantity of muscular fibres and smooth muscle/collagen ratio were found in bladders of castrate rats treated by TRT compared to untreated controls (Tek et al., 2010). These findings suggest that TRT can affect bladder muscle contractility and compliance.

The paradigm that physiologically high levels of testosterone in men account for their increased relative risk for coronary artery disease was challenged at the end of the 20th century. A positive association between androgens deficiency and the presence of coronary artery disease was noted in a case control study comparing men with proven coronary artery disease to men with normal coronary angiograms (English et al., 2000a). Men with coronary artery disease have significantly lower levels of free androgen index and bioavailable testosterone than normal controls (Turhan et al., 2007). Furthermore, low endogenous testosterone concentrations were positively linked to mortality due to CVD (Vermeulen, 2001; Haring et al., 2010; Malkin et al., 2010; Araujo et al., 2011; Corona et al., 2011; Hyde et al., 2012). An increase of $6 \mathrm{nmol} / \mathrm{L}$ serum testosterone, in the Epic-Norfolk study, was associated with an almost 14\% drop in the risk of death (Khaw et al., 2007). Two studies showed that low level of testosterone in young adults were associated with premature coronary artery disease and the number of affected coronary vessels (Turhan et al., 2007; Alkamel et al., 2014). Similarly, serum free testosterone levels were found to be inversely related to carotid atherosclerosis and plaque score (Fukui et al., 2003; Urushiyama et al., 2004).

At an epidemiological level, low testosterone levels are associated with an increased risk for the development of hypertension, obesity, diabetes, metabolic syndrome and ED (Torkler et al., 2011; Garcia-Cruz et al., 2013; Garcia-Cruz et al., 2014). Normalizing testosterone in men with hypogonadism improved all these functions and is associated with a decreased risk of developing heart disease (Bassil et al., 2009; Zitzmann, 2009; Haider et al., 2010; Cai et al., 2014).

TRT has been shown to improve chronic stable angina, severe heart failure, exercise-induced myocardial ischemia and coronary blood flow in patients with coronary heart disease (English et al., 2000b; Borst and Mulligan, 2007; Caminiti et al., 2009). However, two recent published studies found contradictory data (Vigen et al., 2013; Finkle et al., 2014). However, these two studies were 
criticized due to their design, the form of administration of testosterone, and the absence of an initial and/or regular check of haematocrit, dihydrotestosterone, oestrogen and testosterone levels.

\section{Links between Hypogonadism, Prostate Cancer and Metabolic Syndrome/CVD}

Except diabetes, each of the other individual cardiovascular risk factors has been associated with prostate cancer. Men with lower plasma cholesterol concentrations were less likely to develop high grade prostate cancer than men with higher concentrations (Mondul et al., 2010). Obese men were more likely to develop high grade and advanced disease and less likely to develop low grade disease (Parker et al., 2013). An association between active smoking and prostate cancer risk was demonstrated in a meta-analysis of 24 cohort studies (Huncharek et al., 2010). Recently, Ho et al. linked smoking to high grade prostate cancer in the REDUCE trial (Ho et al., 2014).

In contrast, there are considerable evidence inversely associating diabetes and prostate cancer (Bansal et al., 2013). Men with metabolic syndrome scheduled for prostatic biopsy were more likely diagnosed with Gleason score $\geq 7$ (Morote et al., 2013). In a cohort of middle aged Norwegian men followed for 27 years, men with at least three metabolic risk factors had a $56 \%$ increased prostate cancer risk (Lund Haheim et al., 2006). In a prospective population-based study, and during an average follow up of 13 years, the association between metabolic syndrome and risk of prostate cancer was stronger among overweight and obese men with a body mass index $\geq 27 \mathrm{~kg} / \mathrm{m}^{2}$ than in lighter men (Laukkanen et al., 2004). In contrast, an inverse association was found in a community based study in the USA. Metabolic syndrome was associated with an increased prostate cancer risk in African American men but not in White men (Beebe-Dimmer et al., 2009). Considering the possible link between these conditions, one could argue that prevention and treatment of metabolic syndrome could have a positive effect on prostate cancer. Frattaroli et al. indicated in a RCT in prostate cancer men on active surveillance that weight loss was associated with less men progressing to operation at a 2 years follow-up (Frattaroli et al., 2008). A recent meta-analysis demonstrated a significant small prostate cancer risk reduction with physical activity (Liu et al., 2011).

Despite the evidence that statins use does not reduce the risk of prostate cancer, it may selectively decrease the risk of advanced prostate cancer (Olivan et al., 2015). Metaanalyses of metformin and cancer risk in diabetic patients described around one-third reduction in overall cancer risk and cancer mortality in metformin users compared with other antidiabetic drugs (Peairs et al., 2011). However, a large multicentre study did not find an association between metformin use and risk of prostate cancer among older men with diabetes, regardless of cancer grade or method of diagnosis (Margel et al., 2013). In men with pre-existing metabolic syndrome that is at risk for the development of CVD, ADT will adversely affect these conditions. A powerful observational study of a population-based cohort of 73,196 prostate cancer patients treated with androgen ablation therapy has reported an increased risk of incident diabetes, myocardial infarction, coronary heart disease and sudden cardiac death in these men (Keating et al., 2006). Similarly, patients treated with ADT and radiation therapy had higher cardiovascular mortality compared to patients treated by only radiation therapy, observed primarily among men with moderate to severe pre-existing CVD (Dal et al., 2010). Recently, a pooled data from six phase III prospective randomized trials suggested that ADT may be an independent risk factor for CVD (Albertsen et al., 2014). Diabetic men treated with ADT for prostate cancer need more medications to control their glycaemic levels (Morgans et al., 2015). In addition, ADT alters significantly the quality of life of prostate cancer patients due to its associated side effects. These include hot flushes, loss of libido, ED, sexual disturbance, fatigue, anaemia, bone loss and bone fracture, obesity, insulin resistance, lipid alterations, depression and even suicide.

However, the potential association with CV toxicity and death is still controversial. An extensive review of this subject, published recently, noted that CVD is the most common cause of mortality in men with early stage metastatic prostate cancer (Ketchandji et al., 2009). More recently, however, there has been increased interest and research into testosterone supplementation and its relationship to prostate cancer. Several studies have shown that low testosterone level was associated with greater incidence of prostate cancer on biopsy, more aggressive prostate cancer on biopsy and on definitive pathology, and lower probability of abiraterone response in metastatic prostate cancer (Khera et al., 2014). In one study, the probability of detecting prostate cancer was 3.3fold higher in hypogonadal men compared to eugonadal men (Gurbuz et al., 2012). However, testosterone levels were not significantly different between patient with and without prostate cancer and the REDUCE trial demonstrated similar detection rates of prostate cancer among men with low compared with normal baseline testosterone levels (Muller et al., 2012).

Men with high-grade cancer had lower levels of serum testosterone than did those with low-grade cancer and patients with low total testosterone had higher rates of Gleason pattern 4 and extra-capsular extension, increased positive surgical margins and higher body mass index (Botto et al., 2011). In the phase III study COU-AA-301, serum androgen levels at baseline strongly associated with survival and median survival increased with each quartile increase in testosterone level regardless of treatment arm (Ryan et al., 2013). Furthermore, the current literature does not report a statistically significant increase in the development or progression of prostate cancer in men receiving testosterone replacement therapy for symptomatic hypogonadism. This had led some authors to hypothesize the theory of prostate saturation model to explain these results (Morgentaler and Traish, 2009). Normalizing testosterone levels in hypogonadal men can increase prostate size and subsequently elevate PSA levels to normal (Barqawi and Crawford, 2006). Thus, it is hypothesized that TRT normalizes the growth of the prostate by balancing the underdeveloped gland secondary 
to the hypogonadal state. In a novel provocative study, Rhoden and Morgentaler reported no significant change in PSA in 20 patients with pre-treatment PIN and one patient was found to have prostate cancer after biopsy (Rhoden and Morgentaler, 2003). Thus, the authors concluded that men with PIN do not have an increased risk of cancer development when treated with TRT than men without PIN. In a retrospective study, the same authors demonstrated no significant changes in serum PSA and prostate volume in patients treated by TRT for hypogonadism who have prostate cancer under active surveillance (Morgentaler et al., 2011). At a median of 2.5 years of follow-up, TRT was not associated with prostate cancer progression. At present, there has been no evidence demonstrating causal effect of TRT on the development of prostate cancer but the use of TRT in men with a history of prostate cancer is still considered experimental awaiting more randomized controlled trials.

\section{Common Potential Pathophysiology for Health Issues Involving Low Androgen Levels}

Low endogenous testosterone levels have been shown to be associated with each of the above mentioned issues. Androgens are key factors in maintain vascular homeostasis by direct mechanisms involving mainly endothelial cells and smooth muscle cells. At the vascular smooth muscle level, in vivo studies have demonstrated a fast vasodilation effect for testosterone therapy in pulmonary, aortic, mesenteric and coronary vasculature in a variety of species (Yildiz and Seyrek, 2007). Webb et al. reported that short term intracoronary administration of testosterone, at physiological levels, induces coronary artery dilation and increases coronary blood flow in men with coronary artery disease (Webb et al., 1999). Other researchers demonstrated that acute testosterone therapy reduces myocardial ischaemia within $30 \mathrm{~min}$ in men with coronary artery disease (English et al., 2000b).

Pharmacological evidence indicates that non genomic androgen-receptor independent pathways are responsible for the acute testosterone-induced vasodilation effect (Foradori et al., 2008). It involves opening of high conductance voltage sensitive calcium activated potassium channels and inhibition of L-type calcium channels. In addition, in humans, normal androgen levels were demonstrated to inhibit proliferation and intimal migration of vascular smooth muscle cells (Mirone et al., 2009). Another major regulator of vascular homeostasis influenced by testosterone is the endothelium. Impaired endothelial function has been described in both animal and human models as consequence of low circulating testosterone levels (Mirone et al., 2009). Endothelial ultrastructural damages were more common in a group of rats with low endogenous testosterone levels compared to a control group with normal testosterone (Filgueira et al., 2012). Interestingly, a partial degree of recovery was observed after testosterone supplementation. Furthermore, studies have shown that low androgen levels are associated with impairment of proliferation, migration and homing of endothelial progenitor cell as well as with apoptosis of endothelial cells (Mirone et al., 2009). In addition, low testosterone levels were responsible for a pro-inflammatory effect on endothelial cells. Activation of pro-inflammatory cytokines enhances chemotactism and leucocyte infiltration in the arterial wall exacerbating vascular smooth muscle cells apoptosis, endothelial dysfunction, degradation of the fibrin, and plaque rupture (Cutini et al., 2012). In animal models, normalizing testosterone levels has been reported to reduce morphologic markers of atherosclerosis ( $\mathrm{Lu}$ et al., 2007). In humans, replacement of physiologic testosterone levels resulted, through a direct mechanism, in an anti-inflammatory shift in circulating cytokines; interleukin 10 was overexpressed whereas TNF- $\alpha$, interleukin 1- $\beta$ and interleukin 6 were suppressed. All these changes associated with low testosterone levels lead to pathologic structural vascular remodelling such as neointima formation, medial thickening, activation of local inflammatory responses, and impairment of extracellular matrix which are all hallmarks of atherosclerosis and potentiate the pro-thrombotic status (Davies et al., 2005). Furthermore, hypogonadism is increasingly recognized as a component of the metabolic syndrome and share with it the same main mechanism that is insulin resistance and reduced insulin sensitivity. One fourth of type 2 diabetic men have sub-physiologic free testosterone concentrations in association with inappropriately low LH and FSH concentrations (Al Hayek et al., 2013). Animal studies over the last few years have clearly established that insulin action and insulin responsiveness in the brain are necessary for the maintenance of the functional integrity of the hypothalamo-hypophyseal-gonadal axis (Dandona and Dhindsa, 2011). Similarly, in vitro studies have demonstrated that pro-inflammatory cytokines suppress GnRH and LH secretion. A minority of patients have subphysiologic testosterone concentrations with elevated LH and FSH concentrations.

Thus, it is suggested that, in diabetic patients, Leydig cells become also resistant and less testosterone is produced (Beatrice et al., 2014). In addition, high insulin levels associated with insulin resistance inhibit testicular production of testosterone and the hepatic production of SHBG (Kapoor et al., 2005). In obese patients, leptin could also inhibit Leydig cell function (Giovambattista et al., 2003). Young obese insulin resistant men produced less testosterone when stimulated with human choriogonadotropin compared with non-obese men (Pitteloud et al., 2005). Conversely, low androgen levels adversely potentiate individual components of the metabolic syndrome. In the NHANES III study, hypogonadism was found to predict insulin resistance and the future development of type 2 diabetes (Selvin et al., 2007). Low testosterone results in an unfavourable lipid profile with an increase in triglycerides, total cholesterol, LDL, oxidized LDL and lipoprotein-a and a decrease in HDL cholesterol (Haring et al., 2011). These changes are most probably due to increased visceral obesity in men with hypogonadism. Increased adiposity and impairment of metabolic profile could increase blood pressure in these patients. Low testosterone could also independently affect blood pressure.

Several hypothesis exist involving plasma endothelin 1 
levels, nitric oxide synthase, contractile RhoA/Rho-kinase signalling pathway, and asymmetric dimethylarginine (Filgueira et al., 2012). Similarly, cardiovascular risk factors have been shown to be associated with endothelial dysfunction and atherosclerosis. Another important point is that low grade sub-clinical chronic inflammation is involved in the pathogenesis of metabolic syndrome. An overexpression of pro-inflammatory markers and mediators has been reported in smokers and in obese and diabetic patients. Smokers were shown to have significantly elevated serum levels of neutrophils, lymphocytes, monocyte, pro-inflammatory cytokines and serum C-reactive protein when compared to nonsmokers. Leukocyte recruitment mediated by endothelial adhesion molecules are key element for the initiation of atherosclerosis. In parallel, smoking reduces flowmediated dilatation, the earliest marker of endothelial dysfunction and atherosclerosis, in systemic arteries in healthy young adults. Experimental studies demonstrated that cigarette smoke reduces NO bioavailability in the vasculature and increases LDL oxidation that lead to prooxidative state exacerbated by the oxidation catalysing metals in the smoke (McEvoy et al., 2015).

In obese patient, adipose tissue releases a large number of bioactive mediators that influence not only body weight homeostasis but also insulin resistance as well as alterations in lipids, blood pressure, coagulation, fibrinolysis and inflammation, leading to endothelial dysfunction and atherosclerosis as well (Rocha and Libby, 2009). Hyperglycaemia, excess free fatty acid release and insulin resistance induce a several detrimental events in the vascular wall, involving increased endothelial dysfunction and vasoconstriction, oxidative stress, low-grade inflammation and platelet hyperactivity (Purushothaman et al., 2007). This chronic activation of the inflammatory cascade induces a decrease in HDL-cholesterol and phospholipids and an increased accumulation of cholesterol in cells. Furthermore, dyslipidaemia may decrease endothelial activation and affect eicosanoid metabolism leading to an atherogenic environment (Tietge, 2014). Thus each component of the metabolic syndrome could lead by its own to a diffuse endothelial dysfunction and to a low grade chronic proinflammatory state which could be further potentiated by the presence of low testosterone levels.

\section{The Pivotal Role of Atherosclerosis and a Common Pathogenic Cascade}

Hypogonadism and metabolic syndrome are currently well established cardiovascular risk factor leading to atherosclerosis. Nonetheless, atherosclerosis is a diffuse systemic disease process that affects all blood vessels in different tissues leading to chronic hypoxia. However, the onset of symptoms will vary between tissues depending on the artery size and their tolerability. Larger vessels tolerate better the same amount of stenosis compared with the smaller ones. That's why, in the artery size hypothesis, ED precedes coronary artery disease with a time interval of 2-3 years (Jackson et al., 2010). The atherosclerotic rat model had demonstrated that a strong correlation between the increase in mean intima/media thickness, in particular in the distal cavernosal artery, and ED (Arrabal-Polo et al., 2014). The same findings were confirmed recently in a clinical study evaluating intima/media thickness in the cavernosal artery and the carotid artery in men with vasculogenic ED compared to men with ED of other causes (Prezioso et al., 2014). Endothelial dysfunctions, even without definitive arterial stenosis, as well as atherosclerosis with definitive penile artery stenosis represent the main anatomic substrate for vasculogenic ED. A narrowed penile artery lumen decreases the perfusion pressure and arterial flow to the sinusoidal spaces, thus increasing the time to maximal erection and decreasing the rigidity of the erect penis. Tarhan et al. evaluated cavernous oxygen tension in the patients with ED and found a decrease in oxygen tension in corpus cavernosum blood of patients with vasculogenic ED compared to that measured in patients with psychogenic ED (Tarhan et al., 1997). A decrease in oxygen tension may diminish PGE2 that is oxygendependent and subsequently elevate TGF- $\beta 1$-induced collagen synthesis leading to a decrease in cavernous trabecular smooth muscle content and to a diffuse venous leakage as suggested in rabbit and human studies (Rogers et al., 2003; Ryu et al., 2004). Increased deposition of collagen leads to fibrosis and loss of compliance of the cavernosal sinusoid and a diminished smooth muscle content.

Chronic hypoxia leads to an increase in extracellular matrix expansion affecting both interstitium and neural structures of the penis. Increased wall to lumen ratio in the cavernosal arteries contributes to increased peripheral vascular resistance and enhanced vasoconstriction in the penile vasculature (Mirone et al., 2009). Chronic hypoxia contributes to the liberation of local angiotensin II, thromboxane and superoxide that further impair endothelium-dependent relaxation, nerve-evoked relaxation and $\alpha$-adrenergic-stimulated contraction of cavernous muscle aggravating the severity of ED. Furthermore, cavernosal ischemia can induce a dysfunction in the veno-occlusive mechanism as demonstrated in a rabbit model of vasculogenic $\operatorname{ED}$ (Dean and Lue, 2005). In severe cavernosal ischemia a loss or reduction of intercellular communication ion channels and gap junction is seen because of the presence of collagen fibers between cellular membranes (Dean and Lue, 2005).

All these molecular and ultrastructural changes alter the coordinated smooth muscle activity and have a detrimental effect on erectile response.

Chronic induced bladder hypoxia resulting from atherosclerosis is one of the main factors involved in LUTS. It has been demonstrated that pelvic atherosclerosis reduces NO signaling, up-regulates RhoA-ROCK, and is a component of the metabolic syndrome/autonomic hyperactivity. All these mechanisms have been implicated in the development of LUTS but the time course of these effects, are partially elucidated. The complex function of the urothelium and the contractile function of the detrusor depend on adequate supply of oxygen and nutrients from the blood. The most vulnerable part to ischemia is the mucosa, which has a metabolic rate three times higher than 
that of the detrusor. Impairment of neurokinin 2 receptor reactivity and gene expression, increased expression and reactivity of tachykinin positive nerves had been shown in atherosclerosis-induced ischemic rabbit bladder (Azadzoi et al., 2008). It has been hypothesized that these changes could affect the sensitivity of afferent nerves and be responsible for the development of LUTS. Furthermore, during a micturition cycle, bladder blood flow is variable with minimal and maximal blood flow seen during voiding and immediately after micturition, respectively (Yamaguchi et al., 2014). It appears that the ischemia/ reperfusion cycle in ischemic animal bladder models produce significant amount of bladder 5-lipoxygenase, and COX-1 and COX-2 protein expression, and alter leukotriene and PG production (Azadzoi et al., 2008). Neuro-denervation of the bladder and the expression of tissue damaging molecules in the bladder wall depend on the amount of free radicals produced, and the degree and duration of hypoxia. Moderate ischemia may cause detrusor overactivity via sensitization of afferent pathways and post-junctional oversensitivity in response to partial denervation. Progression of denervation and damage to detrusor muscle may cause detrusor underactivity when bladder ischemia becomes severe (Yamaguchi et al., 2014). Moreover, studies in animal models suggest that progressive increase in bladder mass and progressive denervation seen in bladder outlet obstruction could be due to hypoxia (Levin et al., 2003). In addition, clinical studies demonstrated that partial denervation of the detrusor muscle occurs in obstructed human bladder (Speakman et al., 1987).

In this context, some authors had suggested that the therapeutic effect of $\alpha$-adrenergic receptor blockers and PDE5I on male LUTS would be due to the improvement of bladder ischemia. In a clinical study, the bladder capacity and the perfusion pressure increased substantially to reach normal levels after 5 weeks of $\alpha$-adrenergic receptor blocker therapy (Koritsiadis et al., 2010). The potential role of NO/cGMP pathway in the pathophysiology of LUTS/BPH had been recently suggested by the evidence of the anti-proliferative and pro-apoptotic effects of NO donors on culture of smooth muscle tone of bladder, prostate and urethra smooth muscle cells (Nomiya et al., 2013). The reduced function of NO production had been demonstrated in ischemic bladders. Interestingly, in animal studies, PDE5 inhibition restored NO production, reversed RhoA/ROCK contractile signalling and RhoA/ ROCK hyperactivation, and thus prevented deterioration of urodynamic parameters. In animal models of chronic ischemia of the lower urinary tract, administration of PDE5-Is restored normal oxygenation, thereby preventing tissue fibrosis and functional modifications(Nomiya et al., 2013). Pelvic atherosclerosis and abnormal blood flow patterns to the prostate is also implicated in BPH (Berger et al., 2006). Markers of hypoxia are more frequently found in prostatic tissues from patients with BPH compared to normal controls. Several studies found an imbalance in the production/detoxification of reactive oxygen species in BPH resulting in an accumulation of free radicals that cause significant prostate tissue damage (Ghafar et al., 2002). It was postulated that hyperplasia in the stromal and glandular compartments might be induced by stromal growth secondary to hypoxia and that $5 \alpha$-reductase inhibitors reduces prostate size through reducing HIF-1 $\alpha$ and other hypoxia related growth factors (Berger et al., 2003). It was also reported that diabetic patients have significantly worse perfusion rates in the transition zone of the prostate where $\mathrm{BPH}$ arises compared to healthy controls (Berger et al., 2006). Another important point is that these free radicals play a role in the early phase of prostate carcinogenesis. Data from the literature show that high oxidative stress parameters and low antioxidant activity are more prominent in prostate cancer patients compared to BPH patients and normal controls (Khandrika et al., 2009). These oxidative stress species can lead to DNA damage, reduce DNA repair and change the normal regulation of programmed cell death. Human prostate tissue is more vulnerable than other tissues due to more rapid cell turnover and fewer DNA repair enzymes. These species will activate the transcription of many inflammatory cytokines and growth factors and inhibit apoptotic pathway leading to an uncontrolled cell proliferation. Conversely, these chemokines can promote, in turn, the oxidative stress and the genomic instability. Currently, chronic inflammation is regarded as one of the most important enabling characteristics and a principle hallmark of human cancer. The same environmental exposures leading to prostatic inflammation could contribute to the development of prostate cancer.

As already discussed, individual cardiovascular risk factors and diffuse atherosclerotic state are characterized by a chronic state of low grade inflammation. In obese patients, macrophages infiltrate adipose tissues and secrete factors inducing inflammation and lipid accumulation (Perez-Hernandez et al., 2014). Mayi et al. found that the genes expression profile of these adipose tissues infiltrating macrophages in obese patient resemble that of tumor-associated macrophage in cancer patients and differ from monocyte derived macrophage (Mayi et al., 2012). In prostate cancer patients, the genes expression profile of peri-prostatic adipose tissue account for the increased number of deregulated inflammatory cells and reduced immune-surveillance, thereby promoting carcinogenesis. In adjunct to this favorable pro-inflammatory microenvironment, alterations in adipocyte-specific adipokines secretion enhance prostate cancer development and progression in obese patients by increasing insulin resistance and bioavailable IGF-1 and by inhibiting NF- $x$ B (Perez-Hernandez et al., 2014). Another possible mechanisms involved are changes in the sex steroid pathway, and/or the complex biological interaction of hyperglycemia and high insulin levels with prostate tissue but their exact role remains unclear (Perez et al., 2014).

\section{Conclusions}

The above mentioned issues are complex and prevalent disorders in male patients. Their epidemiological association and the possibility of common molecular pathways in their pathogenesis had led to a tremendous shift in their management. Physicians should be aware of the consisting link between these conditions in order 
to screen for low androgen levels and cardiovascular risk factors and to treat in a multidisciplinary approach their patients. A global approach will guarantee a better management with an improvement in men's health and a decrease in their mortality. Although our hypothesis that oxidative stress caused by endothelial dysfunction associated with a dysregulated pro-inflammatory state explains the pathogenesis of each of these conditions, further research is needed to unveil the mysteries of these complex associations.

\section{References}

Al Hayek AA, Khader YS, Jafal S, et al (2013). Prevalence of low testosterone levels in men with type 2 diabetes mellitus: a cross-sectional study. J Family Community Med, 20, 179-86.

Albertsen PC, Klotz L, Tombal B, et al (2014). Cardiovascular morbidity associated with gonadotropin releasing hormone agonists and an antagonist. Eur Urol, 65, 565-73.

Alkamel A, Shafiee A, Jalali A, et al (2014). The association between premature coronary artery disease and level of testosterone in young adult males. Arch Iran Med, 17, 545-50.

Anitha B, Inamadar AC, Ragunatha S (2009). Finasteride-its impact on sexual function and prostate cancer. J Cutan Aesthet Surg, 2, 12-6.

Araujo AB, Dixon JM, Suarez EA, et al (2011). Clinical review: Endogenous testosterone and mortality in men: a systematic review and meta-analysis. J Clin Endocrinol Metab, 96, 3007-19.

Arrabal-Polo MA, Vera-Arroyo B, Lahoz-Garcia C, et al (2014). Erectile dysfunction, metabolic syndrome and arterial disease. Clinical-pathological relation by carotid ultrasonography. Actas Urol Esp, 38, 179-83.

Azadzoi KM, Radisavljevic ZM, Siroky MB (2008). Effects of ischemia on tachykinin-containing nerves and neurokinin receptors in the rabbit bladder. Urol, 71, 979-83.

Bansal D, Bhansali A, Kapil G, et al (2013). Type 2 diabetes and risk of prostate cancer: a meta-analysis of observational studies. Prostate Cancer Prostatic Dis, 16, 151-8.

Barqawi A, Crawford ED (2006). Testosterone replacement therapy and the risk of prostate cancer. Is there a link? Int $J$ Impot Res, 18, 323-8.

Bassil N,Alkaade S, Morley JE (2009). The benefits and risks of testosterone replacement therapy: a review. Prostate Cancer Prostatic Dis, 5, 427-48.

Beatrice AM, Dutta D, Kumar M, et al (2014). Testosterone levels and type 2 diabetes in men: current knowledge and clinical implications. Diabetes Metab Syndr Obes, 7, 481-6.

Beebe-Dimmer JL, Nock NL, Neslund-Dudas C, et al (2009). Racial differences in risk of prostate cancer associated with metabolic syndrome. Urol, 74, 185-90.

Berger AP, Bartsch G, Deibl M, et al (2006). Atherosclerosis as a risk factor for benign prostatic hyperplasia. BJU Int, 98, 1038-42.

Berger AP, Kofler K, Bektic J, et al (2003). Increased growth factor production in a human prostatic stromal cell culture model caused by hypoxia. Prostate, 57, 57-65.

Blumentals WA, Gomez-Caminero A, Joo S, et al (2004). Should erectile dysfunction be considered as a marker for acute myocardial infarction? Results from a retrospective cohort study. Int J Impot Res, 16, 350-3.

Borst SE, Mulligan T (2007). Testosterone replacement therapy for older men. Clin Interv Aging, 2, 561-6.

Botto H, Neuzillet Y, Lebret T, et al (2011). High incidence of predominant Gleason pattern 4 localized prostate cancer is associated with low serum testosterone.J Urol, 186, 1400-5.

Bouwman, II, Blanker MH, Schouten BW, et al (2015). Are lower urinary tract symptoms associated with cardiovascular disease in the Dutch general population? Results from the Krimpen study. World J Urol, 33, 669-76.

Bouwman, II, Van Der Heide WK, Van Der Meer K, et al (2009). Correlations between lower urinary tract symptoms, erectile dysfunction, and cardiovascular diseases: are there differences between male populations from primary healthcare and Urol clinics? A review of the current knowledge. Eur J Gen Pract, 15, 128-35.

Boyle P, Robertson C, Mazzetta C, et al (2003). The association between lower urinary tract symptoms and erectile dysfunction in four centres: the UrEpik study. BJU Int, 92, 719-25.

Braun M, Wassmer G, Klotz T, et al (2000). Epidemiology of erectile dysfunction: results of the 'cologne male survey'. Int J Impot Res, 12, 305-11.

Cai X, Tian Y, Wu T, et al (2014). Metabolic effects of testosterone replacement therapy on hypogonadal men with type 2 diabetes mellitus: a systematic review and metaanalysis of randomized controlled trials. Asian J Androl, 16, 146-52.

Camacho EM, Huhtaniemi IT, O’Neill TW, et al (2013). Ageassociated changes in hypothalamic-pituitary-testicular function in middle-aged and older men are modified by weight change and lifestyle factors: longitudinal results from the European male ageing study. Eur J Endocrinol, 168, 445-55.

Caminiti G, Volterrani M, Iellamo F, et al (2009). Effect of long-acting testosterone treatment on functional exercise capacity, skeletal muscle performance, insulin resistance, and baroreflex sensitivity in elderly patients with chronic heart failure a double-blind, placebo-controlled, randomized study. J Am Coll Cardiol, 54, 919-27.

Chang IH, Oh SY, Kim SC (2009). A possible relationship between testosterone and lower urinary tract symptoms in men. J Urol, 182, 215-20.

Colombo MG, Meisinger C, Amann U, et al (2015). Association of obesity and long-term mortality in patients with acute myocardial infarction with and without diabetes mellitus: results from the MONICA/KORA myocardial infarction registry. Cardiovasc Diabetol, 14, 24.

Corona G, Lee DM, Forti G, et al (2010). Age-related changes in general and sexual health in middle-aged and older men: results from the European male ageing study (EMAS). $J$ Sex Med, 7, 1362-80.

Corona G, Mannucci E, Schulman C, et al (2006). Psychobiologic correlates of the metabolic syndrome and associated sexual dysfunction. Eur Urol, 50, 595-604.

Corona G, Rastrelli G, Monami M, et al (2011). Hypogonadism as a risk factor for cardiovascular mortality in men: a metaanalytic study. Eur J Endocrinol, 165, 687-701.

Couillard C, Gagnon J, Bergeron J, et al (2000). Contribution of body fatness and adipose tissue distribution to the age variation in plasma steroid hormone concentrations in men: the HERITAGE Family Study. J Clin Endocrinol Metab, 85, 1026-31.

Cutini PH, Campelo AE, Agriello E, et al (2012). The role of sex steroids on cellular events involved in vascular disease. J Steroid Biochem Mol Biol, 132, 322-30.

Dal Pra A, Cury FL, Souhami L (2010). Combining radiation therapy and androgen deprivation for localized prostate cancer-a critical review. Curr Oncol, 17, 28-38.

Dandona P, Dhindsa S (2011). Update: Hypogonadotropic hypogonadism in type 2 diabetes and obesity. J Clin Endocrinol Metab, 96, 2643-51. 
Davies JD, Carpenter KL, Challis IR, et al (2005). Adipocytic differentiation and liver $\mathrm{x}$ receptor pathways regulate the accumulation of triacylglycerols in human vascular smooth muscle cells. J Biol Chem, 280, 3911-9.

De Nunzio C, Aronson W, Freedland SJ, et al (2012). The correlation between metabolic syndrome and prostatic diseases. Eur Urol, 61, 560-70.

Dean RC, Lue TF (2005). Physiology of penile erection and pathophysiology of erectile dysfunction. Urol Clin North Am, 32, 379-95.

English KM, Mandour O, Steeds RP, et al (2000a). Men with coronary artery disease have lower levels of androgens than men with normal coronary angiograms. Eur Heart J, 21, 890-4.

English KM, Steeds RP, Jones TH, et al (2000b). Low-dose transdermal testosterone therapy improves angina threshold in men with chronic stable angina: A randomized, doubleblind, placebo-controlled study. Circulat, 102, 1906-11.

Esposito K, Giugliano F, Martedi E, et al (2005). High proportions of erectile dysfunction in men with the metabolic syndrome. Diabetes Care, 28, 1201-3.

Filgueira FP, Lobato NS, DosSantos RA, et al (2012). Endogenous testosterone increases leukocyte-endothelial cell interaction in spontaneously hypertensive rats. Life Sci, 90, 689-94.

Finkle WD, Greenland S, Ridgeway GK, et al (2014). Increased risk of non-fatal myocardial infarction following testosterone therapy prescription in men. PLoS One, $\mathbf{9}, 85805$.

Fisher WA, Rosen RC, Eardley I, et al (2005). Sexual experience of female partners of men with erectile dysfunction: the female experience of men's attitudes to life events and sexuality (FEMALES) study. J Sex Med, 2, 675-84.

Foradori CD, Weiser MJ, Handa RJ (2008). Non-genomic actions of androgens. Front Neuroendocrinol, 29, 169-81.

Frattaroli J, Weidner G, Dnistrian AM, et al (2008). Clinical events in prostate cancer lifestyle trial: results from two years of follow-up. Urol, 72, 1319-23.

Fukui M, Kitagawa Y, Nakamura N, et al (2003). Association between elevated testosterone and development of microalbuminuria during puberty in female subjects with type 1 diabetes: response to Amin et Al. Diabetes Care, 26, 2966-7.

Gacci M, Eardley I, Giuliano F, et al (2011). Critical analysis of the relationship between sexual dysfunctions and lower urinary tract symptoms due to benign prostatic hyperplasia. Eur Urol, 60, 809-25.

Gandaglia G, Briganti A, Jackson G, et al (2014). A systematic review of the association between erectile dysfunction and cardiovascular disease. Eur Urol, 65, 968-78.

Garcia-Cruz E, Leibar-Tamayo A, Romero-Otero J, et al (2014). Marked testosterone deficiency-related symptoms may be associated to higher metabolic risk in men with low testosterone levels. J Sex Med, 11, 2292-301.

Garcia-Cruz E, Leibar-Tamayo A, Romero J, et al (2013). Metabolic syndrome in men with low testosterone levels: relationship with cardiovascular risk factors and comorbidities and with erectile dysfunction. J Sex Med, 10, 2529-38.

Ghafar MA, Puchner PJ, Anastasiadis AG, et al (2002). Does the prostatic vascular system contribute to the development of benign prostatic hyperplasia? Curr Urol Rep, 3, 292-6.

Giovambattista A, Suescun MO, Nessralla CC, et al (2003). Modulatory effects of leptin on leydig cell function of normal and hyperleptinemic rats. Neuroendocrinol, 78, 270-9.

Gurbuz C, Canat L, Atis G, et al (2012). The role of serum testosterone to prostate-specific antigen ratio as a predictor of prostate cancer risk. Kaohsiung J Med Sci, 28, 649-53.
Haider A, Gooren LJ, Padungtod P, et al (2009). Concurrent improvement of the metabolic syndrome and lower urinary tract symptoms upon normalisation of plasma testosterone levels in hypogonadal elderly men. Andrologia, 41, 7-13.

Haider A, Gooren LJ, Padungtod P, et al (2010). Improvement of the metabolic syndrome and of non-alcoholic liver steatosis upon treatment of hypogonadal elderly men with parenteral testosterone undecanoate. Exp Clin Endocrinol Diabetes, 118, 167-71.

Hammarsten J, Hogstedt B, Holthuis N, et al (1998). Components of the metabolic syndrome-risk factors for the development of benign prostatic hyperplasia. Prostate Cancer Prostatic Dis, 1, 157-62.

Haring R, Baumeister SE, Volzke H, et al (2011). Prospective association of low total testosterone concentrations with an adverse lipid profile and increased incident dyslipidemia. Eur J Cardiovasc Prev Rehabil, 18, 86-96.

Haring R, Volzke H, Steveling A, et al (2010). Low serum testosterone levels are associated with increased risk of mortality in a population-based cohort of men aged 20-79. Eur Heart J, 31, 1494-501.

Ho T, Howard LE, Vidal AC, et al (2014). Smoking and risk of low- and high-grade prostate cancer: results from the REDUCE study. Clin Cancer Res, 20, 5331-8.

Holmang S, Marin P, Lindstedt G, et al (1993). Effect of longterm oral testosterone undecanoate treatment on prostate volume and serum prostate-specific antigen concentration in eugonadal middle-aged men. Prostate, 23, 99-106.

Huncharek M, Haddock KS, Reid R, et al (2010). Smoking as a risk factor for prostate cancer: a meta-analysis of 24 prospective cohort studies. Am J Public Health, 100, 693701.

Hyde Z, Norman PE, Flicker L, et al (2012). Low free testosterone predicts mortality from cardiovascular disease but not other causes: the Health in Men Study. J Clin Endocrinol Metab, 97, 179-89.

Isidori AM, Buvat J, Corona G, et al (2014). A critical analysis of the role of testosterone in erectile function: from pathophysiology to treatment-a systematic review. Eur Urol, 65, 99-112.

Isidori AM, Giannetta E, Gianfrilli D, et al (2005). Effects of testosterone on sexual function in men: results of a metaanalysis. Clin Endocrinol, 63, 381-94.

Jackson G, Boon N, Eardley I, et al (2010). Erectile dysfunction and coronary artery disease prediction: evidence-based guidance and consensus. Int J Clin Pract, 64, 848-57.

Kalra S, Agrawal N, Kumar S, et al (2010). Testosterone replacement in male hypogonadism. Clin Pharmacol, 2 , 149-53.

Kapoor D, Aldred H, Clark S, et al (2007). Clinical and biochemical assessment of hypogonadism in men with type 2 diabetes: correlations with bioavailable testosterone and visceral adiposity. Diabetes Care, 30, 911-7.

Kapoor D, Goodwin E, Channer KS, et al (2006). Testosterone replacement therapy improves insulin resistance, glycaemic control, visceral adiposity and hypercholesterolaemia in hypogonadal men with type 2 diabetes. Eur J Endocrinol, 154, 899-906.

Kapoor D, Malkin CJ, Channer KS, et al (2005). Androgens, insulin resistance and vascular disease in men. Clin Endocrinol, 63, 239-50.

Karazindiyanoglu S, Cayan S (2008). The effect of testosterone therapy on lower urinary tract symptoms/bladder and sexual functions in men with symptomatic late-onset hypogonadism. Aging Male, 11, 146-9.

Keating NL, O'Malley AJ, Smith MR (2006). Diabetes and cardiovascular disease during androgen deprivation therapy 
for prostate cancer. J Clin Oncol, 24, 4448-56.

Kenny AM, Kleppinger A, Annis K, et al (2010). Effects of transdermal testosterone on bone and muscle in older men with low bioavailable testosterone levels, low bone mass, and physical frailty. J Am Geriatr Soc, 58, 1134-43.

Ketchandji M, Kuo YF, Shahinian VB, et al (2009). Cause of death in older men after the diagnosis of prostate cancer. $J$ Am Geriatr Soc, 57, 24-30.

Khandrika L, Kumar B, Koul S, et al (2009). Oxidative stress in prostate cancer. Cancer Lett, 282, 125-36.

Khaw KT, Dowsett M, Folkerd E, et al (2007). Endogenous testosterone and mortality due to all causes, cardiovascular disease, and cancer in men: European prospective investigation into cancer in Norfolk (EPIC-Norfolk) prospective population study. Circulat, 116, 2694-701.

Khera M, Crawford D, Morales A, et al (2014). A new era of testosterone and prostate cancer: from physiology to clinical implications. Eur Urol, 65, 115-23.

Kim S, Jeong JY, Choi YJ, et al (2010). Association between lower urinary tract symptoms and vascular risk factors in aging men: the hallym aging Study. Korean J Urol, 51, 477-82.

Kirby M, Chapple C, Jackson G, et al (2013). Erectile dysfunction and lower urinary tract symptoms: a consensus on the importance of co-diagnosis. Int J Clin Pract, 67, 606-18.

Ko YH, Moon du G, Moon KH (2013). Testosterone replacement alone for testosterone deficiency syndrome improves moderate lower urinary tract symptoms: one year follow-up. World J Mens Health, 31, 47-52.

Koritsiadis G, Tyritzis SI, Koutalellis G, et al (2010). The effect of alpha-blocker treatment on bladder hypoxia inducible factor-1 alpha regulation during lower urinary tract obstruction. Int Braz J Urol, 36, 86-94.

Kumar P, Kumar N, ThakurDS, et al (2010). Male hypogonadism: Symptoms and treatment. J Adv Pharm Technol Res, 1, 297301.

Kupelian V, Araujo AB, Chiu GR, et al (2010). Relative contributions of modifiable risk factors to erectile dysfunction: results from the boston area community health (BACH) survey. Prev Med, 50, 19-25.

Laukkanen JA, Laaksonen DE, Niskanen L, et al (2004). Metabolic syndrome and the risk of prostate cancer in Finnish men: a population-based study. Cancer Epidemiol Biomarkers Prev, 13, 1646-50.

Lee RK, Chung D, Chughtai B, et al (2012). Central obesity as measured by waist circumference is predictive of severity of lower urinary tract symptoms. BJU Int, 110, 540-5.

Lee SH, Park TJ, Bae MH, et al (2013). Impact of treatment with statins on prostate-specific antigen and prostate volume in patients with benign prostatic hyperplasia. Korean J Urol, 54, 750-5.

Levin RM, O'Connor LJ, Leggett RE, et al (2003). Focal hypoxia of the obstructed rabbit bladder wall correlates with intermediate decompensation. Neurourol Urodyn, 22, 156-63.

Lin HJ, Weng SF, Yang CM, et al (2013). Risk of hospitalization for acute cardiovascular events among subjects with lower urinary tract symptoms: a nationwide population-based study. PLoS One, 8, 66661.

Liu Y, Hu F, Li D, et al (2011). Does physical activity reduce the risk of prostate cancer? A systematic review and metaanalysis. Eur Urol, 60, 1029-44.

Lu YL, Kuang L, Zhu H, et al (2007). Changes in aortic endothelium ultrastructure in male rats following castration, replacement with testosterone and administration of 5alphareductase inhibitor. Asian J Androl, 9, 843-7.
Lund Haheim L, Wisloff TF, Holme I, et al (2006). Metabolic syndrome predicts prostate cancer in a cohort of middle-aged Norwegian men followed for 27 years. Am J Epidemiol, 164, 769-74.

Malkin CJ, Pugh PJ, Morris PD, et al (2010). Low serum testosterone and increased mortality in men with coronary heart disease. Heart, 96, 1821-5.

Margel D, Urbach D, Lipscombe LL, et al (2013). Association between metformin use and risk of prostate cancer and its grade. J Natl Cancer Inst, 105, 1123-31.

Mayi TH, Daoudi M, Derudas B, et al (2012). Human adipose tissue macrophages display activation of cancer-related pathways. J Biol Chem, 287, 21904-13.

McEvoy JW, Nasir K, DeFilippis AP, et al (2015). Relationship of cigarette smoking with inflammation and subclinical vascular disease: the multi-ethnic study of atherosclerosis. Arterioscler Thromb Vasc Biol, 35, 1002-10.

McVary KT (2005). Erectile dysfunction and lower urinary tract symptoms secondary to BPH. Eur Urol, 47, 838-45.

Mirone V, Imbimbo C, Fusco F, et al (2009). Androgens and morphologic remodeling at penile and cardiovascular levels: a common piece in complicated puzzles? Eur Urol, 56, 309-16.

Mondul AM, Clipp SL, Helzlsouer KJ, et al (2010). Association between plasma total cholesterol concentration and incident prostate cancer in the CLUE II cohort. Cancer Causes Control, 21, 61-8.

Montorsi P, Ravagnani PM, Galli S, et al (2006a). Association between erectile dysfunction and coronary artery disease. Role of coronary clinical presentation and extent of coronary vessels involvement: the COBRA trial. Eur Heart J, 27, 2632-9.

Montorsi P, Ravagnani PM, Galli S, et al (2006b). Association between erectile dysfunction and coronary artery disease: Matching the right target with the right test in the right patient. Eur Urol, 50, 721-31.

Morgans AK, Fan KH, Koyama T, et al (2015). Influence of age on incident diabetes and cardiovascular disease in prostate cancer survivors receiving androgen deprivation therapy. $J$ Urol, 193, 1226-31.

Morgentaler A, Lipshultz LI, Bennett R, et al (2011). Testosterone therapy in men with untreated prostate cancer. J Urol, 185, 1256-60.

Morgentaler A, Traish AM (2009). Shifting the paradigm of testosterone and prostate cancer: the saturation model and the limits of androgen-dependent growth. Eur Urol, 55, 310-20.

Morote J, Ropero J, Planas J, et al (2013). Metabolic syndrome increases the risk of aggressive prostate cancer detection. BJU Int, 111, 1031-6.

Muller RL, Gerber L, Moreira DM, et al (2012). Serum testosterone and dihydrotestosterone and prostate cancer risk in the placebo arm of the reduction by dutasteride of prostate cancer events trial. Eur Urol, 62, 757-64.

Nehra A, Jackson G, Miner M, et al (2012). The Princeton III Consensus recommendations for the management of erectile dysfunction and cardiovascular disease. Mayo Clin Proc, 87, 766-78.

$\mathrm{Ng} \mathrm{CF}$, Wong A, Li ML, et al (2007). The prevalence of cardiovascular risk factors in male patients who have lower urinary tract symptoms. Hong Kong Med J, 13, 421-6.

Nock MK, Borges G, Bromet EJ, et al (2008). Suicide and suicidal behavior. Epidemiol Rev, 30, 133-54.

Nomiya M, Burmeister DM, Sawada N, et al (2013). Prophylactic effect of tadalafil on bladder function in a rat model of chronic bladder ischemia. J Urol, 189, 754-61.

Olivan M, Rigau M, Colas E, et al (2015). Simultaneous treatment with statins and aspirin reduces the risk of prostate 
cancer detection and tumorigenic properties in prostate cancer cell lines. Biomed Res Int, 2015, 762178.

Ozden C, Ozdal OL, Urgancioglu G, et al (2007). The correlation between metabolic syndrome and prostatic growth in patients with benign prostatic hyperplasia. Eur Urol, 51, 199-203.

Paans N, van der Veen WJ, van der Meer K, et al (2011). Time spent in primary care for hip osteoarthritis patients once the diagnosis is set: a prospective observational study. $B M C$ Fam Pract, 12, 48.

Parker AS, Thiel DD, Bergstralh E, et al (2013). Obese men have more advanced and more aggressive prostate cancer at time of surgery than non-obese men after adjusting for screening PSA level and age: results from two independent nested casecontrol studies. Prostate Cancer Prostatic Dis, 16, 352-6.

Parsons JK (2010). Benign prostatic hyperplasia and male lower urinary tract symptoms: epidemiology and risk factors. Curr Bladder Dysfunct Rep, 5, 212-8.

Parsons JK, Kashefi C (2008). Physical activity, benign prostatic hyperplasia, and lower urinary tract symptoms. Eur Urol, 53, 1228-35.

Parsons JK, Messer K, White M, et al (2011). Obesity increases and physical activity decreases lower urinary tract symptom risk in older men: the Osteoporotic Fractures in Men study. Eur Urol, 60, 1173-80.

Peairs KS, Barone BB, Snyder CF, et al (2011). Diabetes mellitus and breast cancer outcomes: a systematic review and metaanalysis. J Clin Oncol, 29, 40-6.

Pearl JA, Berhanu D, Francois N, et al (2013). Testosterone supplementation does not worsen lower urinary tract symptoms. J Urol, 190, 1828-33.

Perez-Hernandez AI, Catalan V, Gomez-Ambrosi J, et al (2014). Mechanisms linking excess adiposity and carcinogenesis promotion. Front Endocrinol, 5, 65.

Pitteloud N, Hardin M, Dwyer AA, et al (2005). Increasing insulin resistance is associated with a decrease in Leydig cell testosterone secretion in men.J Clin Endocrinol Metab, 90, 2636-41.

Prezioso D, Iacono F, Russo U, et al (2014). Evaluation of penile cavernosal artery intima-media thickness in patients with erectile dysfunction. A new parameter in the diagnosis of vascular erectile dysfunction. Our experience on 59 cases. Arch Ital Urol Androl, 86, 9-14.

Purushothaman KR, Meerarani P, Moreno PR (2007). Inflammation and neovascularization in diabetic atherosclerosis. Indian J Exp Biol, 45, 93-102.

Rhoden EL, Morgentaler A (2003). Testosterone replacement therapy in hypogonadal men at high risk for prostate cancer: results of 1 year of treatment in men with prostatic intraepithelial neoplasia. J Urol, 170, 2348-51.

Rocha VZ, Libby P (2009). Obesity, inflammation, and atherosclerosis. Nat Rev Cardiol, 6, 399-409.

Roehrborn CG, Barkin J, Siami P, et al (2011). Clinical outcomes after combined therapy with dutasteride plus tamsulosin or either monotherapy in men with benign prostatic hyperplasia (BPH) by baseline characteristics: 4-year results from the randomized, double-blind Combination of Avodart and Tamsulosin (CombAT) trial. BJU Int, 107, 946-54.

Rogers RS, Graziottin TM, Lin CS, et al (2003). Intracavernosal vascular endothelial growth factor (VEGF) injection and adeno-associated virus-mediated VEGF gene therapy prevent and reverse venogenic erectile dysfunction in rats. Int J Impot Res, 15, 26-37.

Rohrmann S, Smit E, Giovannucci E, et al (2005). Association between markers of the metabolic syndrome and lower urinary tract symptoms in the Third National Health and Nutrition Examination Survey (NHANES III). Int J Obes, 29,310-6.
Rosen R, Altwein J, Boyle P, et al (2003). Lower urinary tract symptoms and male sexual dysfunction: the multinational survey of the Aging Male (MSAM-7). Eur Urol, 44, 637-49.

Rosen RC, Fisher WA, Eardley I, et al (2004). The multinational Men's Attitudes to Life Events and Sexuality (MALES) study: I. Prevalence of erectile dysfunction and related health concerns in the general population. Curr Med Res Opin, 20, 607-17.

Ryan CJ, Molina A, Li J, et al (2013). Serum androgens as prognostic biomarkers in castration-resistant prostate cancer: results from an analysis of a randomized phase III trial. $J$ Clin Oncol, 31, 2791-8.

Ryu JK, Han JY, Chu YC, et al (2004). Expression of cavernous transforming growth factor-beta1 and its type II receptor in patients with erectile dysfunction. Int $J$ Androl, 27, 42-9.

Schatzl G, Brossner C, Schmid S, et al (2000). Endocrine status in elderly men with lower urinary tract symptoms: correlation of age, hormonal status, and lower urinary tract function. the prostate study group of the austrian society of urology. Urol, 55, 397-402.

Seftel AD, de la Rosette J, Birt J, et al (2013). Coexisting lower urinary tract symptoms and erectile dysfunction: a systematic review of epidemiological data. Int J Clin Pract, 67, 32-45.

Selvin E, Feinleib M, Zhang L, et al (2007). Androgens and diabetes in men: results from the Third National Health and Nutrition Examination Survey (NHANES III). Diabetes Care, 30, 234-8.

Shigehara K, Sugimoto K, Konaka H, et al (2011). Androgen replacement therapy contributes to improving lower urinary tract symptoms in patients with hypogonadism and benign prostate hypertrophy: a randomised controlled study. Aging Male, 14, 53-8.

Shiri R, Hakkinen J, Koskimaki J, et al (2007). Erectile dysfunction influences the subsequent incidence of lower urinary tract symptoms and bother. Int J Impot Res, 19, 317-20.

Siegel R, Naishadham D, Jemal A (2012). Cancer statistics, 2012. CA Cancer J Clin, 62, 10-29.

Sih R, Morley JE, Kaiser FE, et al (1997). Testosterone replacement in older hypogonadal men: a 12-month randomized controlled trial. J Clin Endocrinol Metab, 82, 1661-7.

Speakman MJ, Brading AF, Gilpin CJ, et al (1987). Bladder outflow obstruction--a cause of denervation supersensitivity. J Urol, 138, 1461-6.

St Sauver JL, Jacobsen SJ, Jacobson DJ, et al (2011). Statin use and decreased risk of benign prostatic enlargement and lower urinary tract symptoms. BJU Int, 107, 443-50.

Tarhan F, Kuyumcuoglu U, Kolsuz A, et al (1997). Cavernous oxygen tension in the patients with erectile dysfunction. Int J Impot Res, 9, 149-53.

Tek M, Balli E, Cimen B, et al (2010). The effect of testosterone replacement therapy on bladder functions and histology in orchiectomized mature male rats. Urol, 75, 886-90.

Thom T, Haase N, Rosamond W, et al (2006). Heart disease and stroke statistics--2006 update: a report from the American Heart Association Statistics Committee and Stroke Statistics Subcommittee. Circulat, 113, 85-151.

Tietge UJ (2014). Hyperlipidemia and cardiovascular disease: inflammation, dyslipidemia, and atherosclerosis. Curr Opin Lipidol, 25, 94-5.

Torkler S, Wallaschofski H, Baumeister SE, et al (2011). Inverse association between total testosterone concentrations, incident hypertension and blood pressure. Aging Male, 14, 176-82.

Turhan S, Tulunay C, Gulec S, et al (2007). The association between androgen levels and premature coronary artery 
disease in men. Coron Artery Dis, 18, 159-62.

Urushiyama T, Akutsu S, Miyazaki J, et al (2004). Change from a hard to soft diet alters the expression of insulin-like growth factors, their receptors, and binding proteins in association with atrophy in adult mouse masseter muscle. Cell Tissue Res, 315, 97-105.

Vermeulen A (2001). Androgen replacement therapy in the Aging Male--a critical evaluation. J Clin Endocrinol Metab, 86, 2380-90.

Vigen R, O'Donnell CI, Baron AE, et al (2013). Association of testosterone therapy with mortality, myocardial infarction, and stroke in men with low testosterone levels. JAMA, 310, 1829-36.

Vlachopoulos C,Aznaouridis K, Stefanadis C (2010). Prediction of cardiovascular events and all-cause mortality with arterial stiffness: a systematic review and meta-analysis. J Am Coll Cardiol, 55, 1318-27.

Vlachopoulos C, Jackson G, Stefanadis C, et al (2013a). Erectile dysfunction in the cardiovascular patient. Eur Heart J, 34, 2034-46.

Vlachopoulos C, Rokkas K, Ioakeimidis N, et al (2005). Prevalence of asymptomatic coronary artery disease in men with vasculogenic erectile dysfunction: a prospective angiographic study. Eur Urol, 48, 996-1002.

Vlachopoulos CV, Terentes-Printzios DG, Ioakeimidis NK, et al (2013b). Prediction of cardiovascular events and all-cause mortality with erectile dysfunction: a systematic review and meta-analysis of cohort studies. Circ Cardiovasc Qual Outcomes, 6, 99-109.

Wang C, Nieschlag E, Swerdloff RS, et al (2009). ISA, ISSAM, EAU, EAA and ASA recommendations: investigation, treatment and monitoring of late-onset hypogonadism in males. Aging Male, 12, 5-12.

Webb CM, McNeill JG, Hayward CS, et al (1999). Effects of testosterone on coronary vasomotor regulation in men with coronary heart disease. Circulat, 100, 1690-6.

Wehrberger C, Temml C, Gutjahr G, et al (2011). Is there an association between lower urinary tract symptoms and cardiovascular risk in men? A cross sectional and longitudinal analysis. Urol, 78, 1063-7.

Wein AJ, Coyne KS, Tubaro A, et al (2009). The impact of lower urinary tract symptoms on male sexual health: EpiLUTS. BJU Int, 103, 33-41.

Yamaguchi O, Nomiya M, Andersson KE (2014). Functional consequences of chronic bladder ischemia. Neurourol Urodyn, 33, 54-8.

Yildiz O, Seyrek M (2007). Vasodilating mechanisms of testosterone. Exp Clin Endocrinol Diabetes, 115, 1-6.

Zitzmann M (2009). Testosterone deficiency, insulin resistance and the metabolic syndrome. Nat Rev Endocrinol, 5, 673-81. 